This document is confidential and is proprietary to the American Chemical Society and its authors. Do not copy or disclose without written permission. If you have received this item in error, notify the sender and delete all copies.

\title{
Photo-Transformation Trajectories of Nitro-Spiropyran in Hybrid Compounds with [60]Fullerene
}

\begin{tabular}{|r|l|}
\hline Journal: & The Journal of Physical Chemistry \\
\hline Manuscript ID & jp-2019-04197e.R1 \\
\hline Manuscript Type: & Article \\
\hline Date Submitted by the & n/a \\
\hline Complete List of Authors: & $\begin{array}{l}\text { Pomogaev, Vladimir; Kyungpook National University, Department of } \\
\text { Chemistry and Green-Nano Materials Research Center, College of Natural } \\
\text { Sciences; Tomskij gosudarstvennyj universitet, Physics, } \\
\text { Avramov, Pavel; Kyungpook National University, Department of } \\
\text { Chemistry } \\
\text { Ruud, Kenneth; UiT Norges arktiske universitet, Center for Theoretical } \\
\text { and Cmputational Chemistry }\end{array}$ \\
\hline
\end{tabular}

\section{SCHOLARONE ${ }^{\text {m }}$} Manuscripts 


\title{
Photo-Transformation Trajectories of Nitro- Spiropyran in Hybrid Compounds with [60]Fullerene
}

\author{
Vladimir A. Pomogaev, ${ }^{*} \dagger$ Pavel V. Avramov, ${ }^{\square}$ Kenneth Ruud \\ $\square$ Department of Chemistry and Green-Nano Materials Research Center, College of Natural \\ Sciences. Kyungpook National University 1370 Sankyuk-dong, Buk-gu, Daegu, 702-701, \\ Republic of Korea. \\ ${ }^{\dagger}$ Tomsk State University, 36 Lenin Prospekt, Tomsk, Russian Federation \\ $\$$ Hylleraas Centre for Quantum Molecular Sciences, Department of Chemistry, University of \\ Tromsø-The Arctic University of Norway, N-9037 Tromsø, Norway
}

*Corresponding Author Email: helperv@gmail.com

and helper@knu.ac.kr 


\section{ABSTRACT}

Photo- and thermo- isomerization trajectories of various conversion pathways between nitrospiropyran and its trans-trans-cis merocyanine form were produced and the role of $n \pi *$ states was investigated along the corresponding potential energy surface calculated using the $\omega$ B97XD functional using the cc-pVDZ basis set. The nondissociative $n \pi^{*}$ states on the photo-isomerization trajectories can switch from/to the dissociative photoactive $\pi \pi^{*}$ state at two intersections between their energy surfaces. The photochromic properties inherited in hybrid compounds of nitrospiropyran-containing [60]fullerene is interpreted as a reversible "dual energy bypass" $\pi \pi^{*} \leftrightarrow n \pi^{*}$ mechanism in terms of both adiabatic absorption and highly effective nonadiabatic dissipative transitions between the excited states of the photochromic fragment, that prevents energy loss through the [60]fullerene and in this way keep the photochromic properties intact.

\section{INTRODUCTION}

The photochromism of organic molecules such as spiropyran derivatives have been used as basic elements of smart switches. ${ }^{1-8}$ These switching processes are initiated by light-driven interconversion between their colorless "ring closed" and colored "ring open" forms. In particular, the nitro-substituted spiropyran 1',3',3'-trimethyl-6-nitro-spiro[2H-1-benzopyran-2,2'-indoline] (nSP) undergoes bond cleavage of the spiro motif $\left(\mathrm{C}_{\text {spiro }}-\mathrm{O}\right)$ by means of a photochromic conversion ( $\mathrm{s} 2 \mathrm{~m}$ ), being stabilized in the zwitterionic nitro-merocyanine (nMC) form. ${ }^{9-13}$ The most stable trans-trans-cis (TTC) merocyanine conformer is formed through $E$-isomerization around 
the spiro- and pyran-bridging double bond. ${ }^{14-16}$ All possible isomers can be defined by the cis/trans orientation around each of the $\alpha, \beta$ and $\gamma$ bonds that correspond to the stereochemical $\left(\mathrm{N}_{1^{\prime}}, \mathrm{C}_{\text {spiro }}\right)^{\wedge}\left(\mathrm{C}_{3}, \mathrm{C}_{4}\right), \quad\left(\mathrm{C}_{\text {spiro }}, \mathrm{C}_{3}\right)^{\wedge}\left(\mathrm{C}_{4}, \mathrm{C}_{4 \mathrm{a}}\right)$ and $\left(\mathrm{C}_{3}, \mathrm{C}_{4}\right)^{\wedge}\left(\mathrm{C}_{4 \mathrm{a}}, \mathrm{C}_{8 \mathrm{a}}\right)$ dihedral torsion angles, respectively ${ }^{17-19}$ (Figure 1).

Recent work ${ }^{20}$ on the photochromic properties of spiropyrans in hybrid compounds has been limited to comparisons of absorption characteristics of nSP and halogenated spiropyrans containing the pyrrolidino[60]fullerene $\left(\mathrm{PF}_{60}\right)$. The absorption by merocyanine

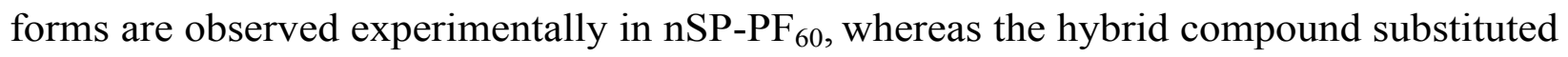
by $\mathrm{Cl}$ or $\mathrm{F}$ atoms do not display photochromism..$^{21,22}$

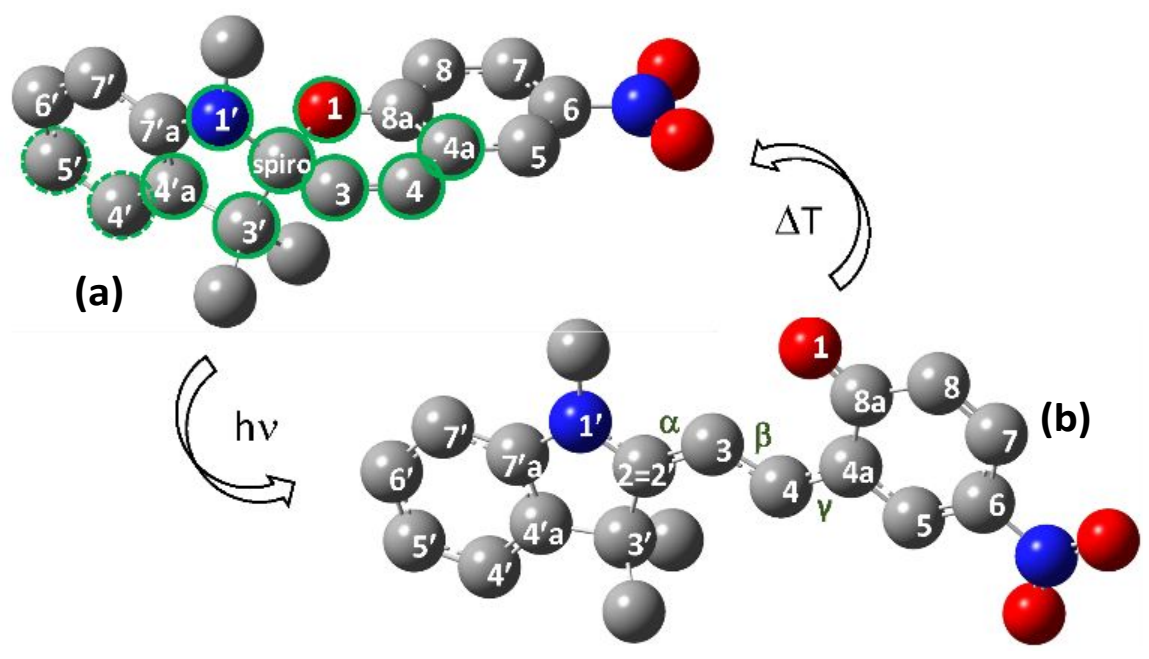

Figure 1. Structure and accepted standard numbering of nSP (a) and nMC (b) where green circle lines denote atoms which contribute the most to the difference between the ground $S_{G,|0,0\rangle}$ and excited $S_{\pi \pi, 1,0\rangle}$ configurations. Dashed green lines in (a) define the bond about which the sequential torsions are being explored.

The low-lying $n \pi^{*}$ states formed by the lone pairs of the nitro substituents was proposed to change the photochromic mechanism of the dissipative excited-state through the $\mathrm{S}_{\pi \pi}(n \mathrm{SP}) \rightarrow$ 
intermediate $n \pi^{*}$ states $\rightarrow \mathrm{S}_{\pi \pi}(n \mathrm{MC})$ channel for photo-transformation of the corresponding hybrid compound..$^{20}$ This conclusion was based on an analysis of the electronic structure of equilibrated $\mathrm{nSP}$ and nMC conformers in the ground and excited electronic states. However, neither kinetic photoinduced excited-state trajectories of the $\mathrm{C}_{\text {spiro }}-\mathrm{O}$ dissociation nor the $\mathrm{s} 2 \mathrm{~m}$ transformation were considered in previous work. A detailed investigation of the role of the $n \pi *$ states in the photochromic conversion along the photo-isomerization trajectory (PIT) is therefore needed.

Different photochemical and thermal kinetic mechanisms have been proposed in previous studies, where transition states $(\mathrm{TS})^{14,17,23}$ and/or conical intersections $(\mathrm{CI})^{18,19,24}$ between the $\pi \rightarrow \pi^{*}$ excited and ground electronic states were calculated in order to identify both the first reaction intermediate and the final product. All these studies only considered unsubstituted or even reduced models of spiropyran. Moreover, the photochemical processes involve the electronic ground state through the rate of $\mathrm{C}_{\text {spiro }}-\mathrm{O}$ bond cleavage in the pre-transformation phase, estimated to be about $k_{\mathrm{tr}} \sim 10^{12} \mathrm{~s}^{-1}$, which exceeds the emission rate of $k_{\mathrm{r}} \sim 10^{8} \mathrm{~s}^{-1}$ and competes with the vibrational relaxation processes $k_{\mathrm{vr}}=10^{12}-10^{14}$ $\mathrm{s}^{-1} \cdot 19,24-28$ The non-adiabatic $\mathrm{s} 2 \mathrm{~m}$ conversion proceeds through a series of consecutive excited vibrational-electronic (vibronic) states originating from vertical adiabatic absorption at the spiropyran moiety.

The present theoretical study aims to shed light on the excited quasi-stationary electronic states that are involved in PIT. State-of-the-art quantum-chemical calculations are carried out in order to study the potential-energy surface (PES) of the photochemical processes between $n \mathrm{SP}$ and $n \mathrm{MC}$ to obtain detailed insight into the role of the nitro substituent for the efficiency of the photochromic transformation. 


\section{COMPUTATIONAL DETAILS}

Both the ground and the excited states of $n \mathrm{SP}$ and $n \mathrm{MC}$ as well as [60]fullerene and the bridges between them were optimized separately and partially optimized in the transformation processes (vide infra), by time-dependent density functional theory (TDDFT) using Gaussian 09. ${ }^{29}$ The hybrid range-separated Generalized Gradient Approximation (GGA) XC-functional, $\omega \mathrm{B} 97 \mathrm{XD},{ }^{30,31}$ in combination with Dunning's correlation-consistent double-zeta basis set, cc-pVDZ, ${ }^{32}(\omega \mathrm{B} 97 \mathrm{XD} / \mathrm{cc}-\mathrm{pVDZ})$ was used as in previous work. ${ }^{20}$ All electronic structure calculations were performed using the same combination of functional and basis set. It was established ${ }^{24}$ that $\omega \mathrm{B} 97 \mathrm{XD} / \mathrm{cc}-\mathrm{pVDZ}$ provides overall the most consistent ground- and excited-state optimized geometries compared to the second-order coupled-cluster approximation with resolution-of-theidentity (RI-CC2). ${ }^{33}$

To consider the intrinsic kinetic mechanism of the s2m conversion, the role of solvent effects on spectral shifts and the relation between the rates of dissipative emission and radiationless transitions are not included in the calculations. The mechanism is defined by the electronic properties of the chemical structures while any external perturbation is assumed as a correction to the main process.

The relative energies $E[\mathrm{kcal} / \mathrm{mol}]$ of the conformers were obtained at each point on the isomerization trajectories as the differences between the total electronic ground- or excited-state energies of a specific conformer and the minimum total energy of the optimized $n \mathrm{SP}$ structure in 
the electronic ground state. PESs of the PITs were produced through partial optimizations in two stages. One geometric parameter was fixed at each point of the trajectory. The first step was to reach a quasi-stationary conformer (QSC) in the excited state by first fixing the $\mathrm{C}_{\text {spiro.. }} \mathrm{O}$ length followed by a gradual elongation of the bond for each additional point on an isomerization trajectory, optimizing the rest of the structure in its excited state. Second, the $\beta$ dihedral angle was fixed to find a minimum energy barrier for the transformation between QSC and the final equilibrated $n \mathrm{MC}$ in the excited state. The PESs of the thermal ${ }^{14,20}$ back-conversion to $n \mathrm{SP}$ was obtained by relaxing the oxygen-spiro distance in the electronic ground state.

\section{RESULTS AND DISSCUSSION}

Ground- and excited-state structures. An excitation of the spiropyrans leads to an unstable compound and a breaking of the ring-opening $\mathrm{C}_{\text {spiro }}-\mathrm{O}$ bond, which prevents fluorescence relaxation (in particular to $\mathrm{nSP}) \cdot{ }^{20,24}$

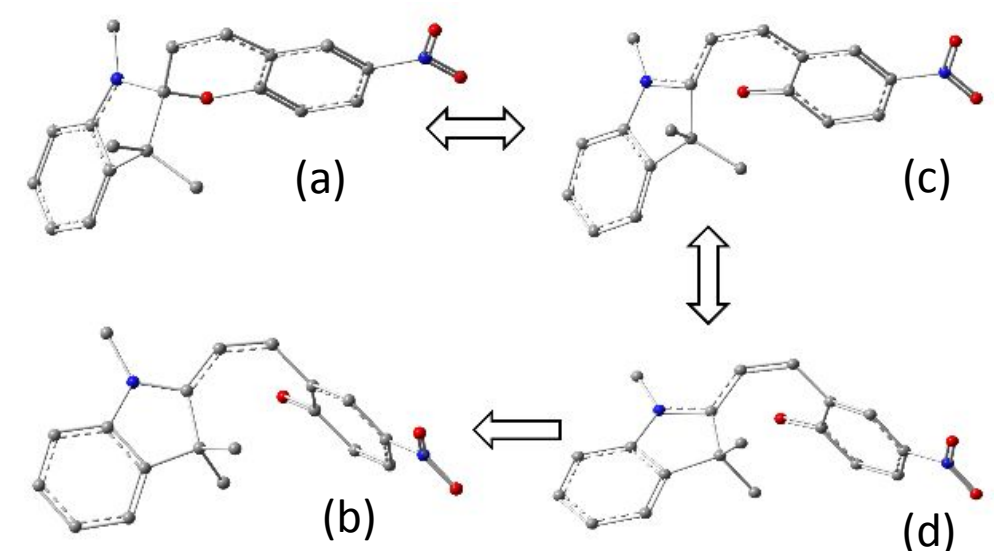

Figure 2. $\mathrm{C}_{\text {spiro.. }} \mathrm{O}$ distance elongation trajectory from equilibrium structure (a) optimized to QSC in $\mathrm{S}_{\pi \pi}$ with the length $=4.05 \AA$ (b) through an intermediate conformer with fixed $\left|\mathrm{C}_{\text {spiro }}-\mathrm{O}\right|=2.00 \AA$ (c) to the energy barrier of no-return point $\left|\mathrm{C}_{\text {spiro. }} \mathrm{O}\right|=3.00 \AA$ (d). 
The part of the compound most sensitive to configurational change in the excited states is the spiro unit at the intersection between the dihydropyrrole and dihydropyran motives (Figure 1). These changes are the dominating causes of the observed spectral shifts due to significant redistribution of electronic density on the segment that leads to the breaking of the oxy-spiro bond.

Table 1. Key geometric parameters ${ }^{34,35}$ of $n$ SPs optimized in the ground (upper line) and $S_{\pi \pi}$ (lower line) excited states (see Figure 1).

\begin{tabular}{|c|c|c|c|c|c|c|c|}
\hline param. $^{\text {atom* }}$ & $\mathbf{C}_{\mathbf{3}^{\prime}}$ & $\mathbf{C}_{2, \text { spiro }}$ & $\mathbf{N}_{1^{\prime}}$ & $\mathbf{C}_{3}$ & $\mathrm{C}_{4}$ & $\mathbf{C}_{4 a}$ & $\mathbf{O}_{1}$ \\
\hline length, & 1.516 & 1.574 & 1.440 & 1.502 & 1.336 & 1.459 & 1.464 \\
\hline$[\AA]$ & 1.501 & 1.583 & 1.513 & 1.475 & 1.403 & 1.409 & 1.422 \\
\hline \multirow{2}{*}{$\begin{array}{l}\text { bend, } \\
\text { [degree] }\end{array}$} & 130.9 & 100.2 & 103.5 & 114.7 & 122.7 & 120.5 & 108.1 \\
\hline & 131.0 & 102.0 & 101.3 & 119.0 & 18.0 & 120.8 & 108.5 \\
\hline \multirow{2}{*}{$\begin{array}{l}\text { torsion, } \\
\text { [degree] }\end{array}$} & 177.6 & 164.1 & 28.8 & 151.6 & 105.9 & 3.6 & -83.5 \\
\hline & 175.8 & 165.2 & 22.4 & 145.3 & 100.0 & 13.4 & -81.7 \\
\hline
\end{tabular}

The photo-instability of $n \mathrm{SP}$ is caused by the dissociative nature of the excited states, making it impossible to determine any stationary points on the excited-state PES. Nevertheless, depending on the initial conditions and the choice of computational method, stationary points may be observed computationally. In particular, the dissociative nature of the excited $n \mathrm{SP}$ strongly depends upon the choice of functional and basis set used in the TD-DFT calculation. In the active $\pi \pi^{*}$ excited state, the molecule will spontaneously relax to an intermediate QSC with a broken $\mathrm{C}_{\text {spiro }}-\mathrm{O}$ bond, similar to (Figure 2d) the first stationary point in the $s 2 m$ transformation. ${ }^{20}$ In contrast, $\omega \mathrm{B} 97 \mathrm{XD} / \mathrm{cc}-\mathrm{pVDZ}$ led to the lowest photoactive excited state where the $\mathrm{C}_{\text {spiro }}-\mathrm{O}_{1}$ bond 
length is even slightly shorter (1.422 $\AA$ vs $1.464 \AA$ ) than in the ground state of the molecule (Table 1).

The bond lengths as well as the corresponding bending and dihedral angles that display the largest changes in the spiro unit are collected in Table 1 (more details in SI), where dihedral torsions originating at $\mathrm{C}_{5^{\prime}}$ and following the covalent bonds as well as relevant bending angles originating from $\mathrm{C}_{4^{\prime}}$ are reported for the ground and excited state. The structural changes can provide an understanding of the photochromic conversion and the rate of this process significantly exceeds nSP emission from its excited state. Despite the slightly shorter $\mathrm{C}_{\text {spiro }}-\mathrm{O}_{1}$ bond length in the excited configuration at the $\omega \mathrm{B} 97 \mathrm{XD} / \mathrm{cc}-\mathrm{pVDZ}$ level of theory, the dihedral torsion around the $\mathrm{C}_{3}-\mathrm{C}_{4}$ bond, corresponding to the $\beta$ orientation in the merocyanine classification, increases by $10^{\circ}$ toward $\mathrm{nMC}$. The largest distortions of the bending angles for $\mathrm{C}_{3}$ and $\mathrm{C}_{4}$ are about $4-5^{\circ}$ in the dihydropyran ring that is going to open. The other bending angles do not change by more than $2^{\circ}$.

Table 2. Dihedral torsion angles $\left[^{\circ}\right]$ in ground (GS) and existed (ex) states.

\begin{tabular}{cccccc}
\hline angle $^{\text {conf. }}$ & $n \mathrm{SPGS}$ & $n \mathrm{SP}^{\mathrm{ex}}$ & $\mathrm{QSC}$ & $n \mathrm{MC}^{\mathrm{ex}}$ & $n \mathrm{MC}^{\mathrm{GS}}$ \\
\hline$\alpha$ & -136.2 & -142.5 & -172.2 & -167.4 & 180.0 \\
$\beta$ & 3.6 & 13.4 & 5.9 & 175.5 & 180.0 \\
$\gamma$ & 6.8 & 1.5 & 86.9 & -27.1 & 0.0 \\
\hline
\end{tabular}

The $\alpha, \beta$ and $\gamma$ dihedral angles are the geometric parameters which most strongly define the conformations, and they are compared for $n \mathrm{SP}$ and $n \mathrm{MC}$ in the ground and excited states as well as the QSC on the photoisomerization trajectory in Table 2. The plain $n \mathrm{MC}$ structure of the ground state is significantly distorted upon excitation. The main difference between the $n \mathrm{SP}$ and $n \mathrm{MC}$ is 
in the twist around the $\beta$ bond. The main QSC feature is the $\gamma$ angle close to $90^{\circ}$ that is a major obstacle for the conversion between the initial and final isomerized forms.

Adiabatic and non-adiabatic conversion processes. To study the kinetics of the $\mathrm{s} 2 \mathrm{~m}$ transformation, the adiabatic vertical excitation $G S \rightarrow S_{|\pi \pi(\mathrm{nSP}, \mathrm{FC})\rangle}$ from the ground vibrational state of the electronic ground state $G S \equiv S_{|0,0\rangle}$ to the photoactive Frank-Condon (FC) $\pi \rightarrow \pi^{*}$ state should be determined (at $\omega \mathrm{B} 97 \mathrm{XD} / \mathrm{cc}-\mathrm{pVDZ}$ level of theory, the relative energy of the excited state is $E$ $=102 \mathrm{kcal} / \mathrm{mol})$. A schematics of the consecutive adiabatic/non-adiabatic transitions of $G S \rightarrow S_{|\pi \pi(\mathrm{nSP}, \mathrm{FCo})\rangle} \rightarrow S_{|n \pi(\mathrm{nSP}, \mathrm{FCo})\rangle}$ is presented in Figure 3.

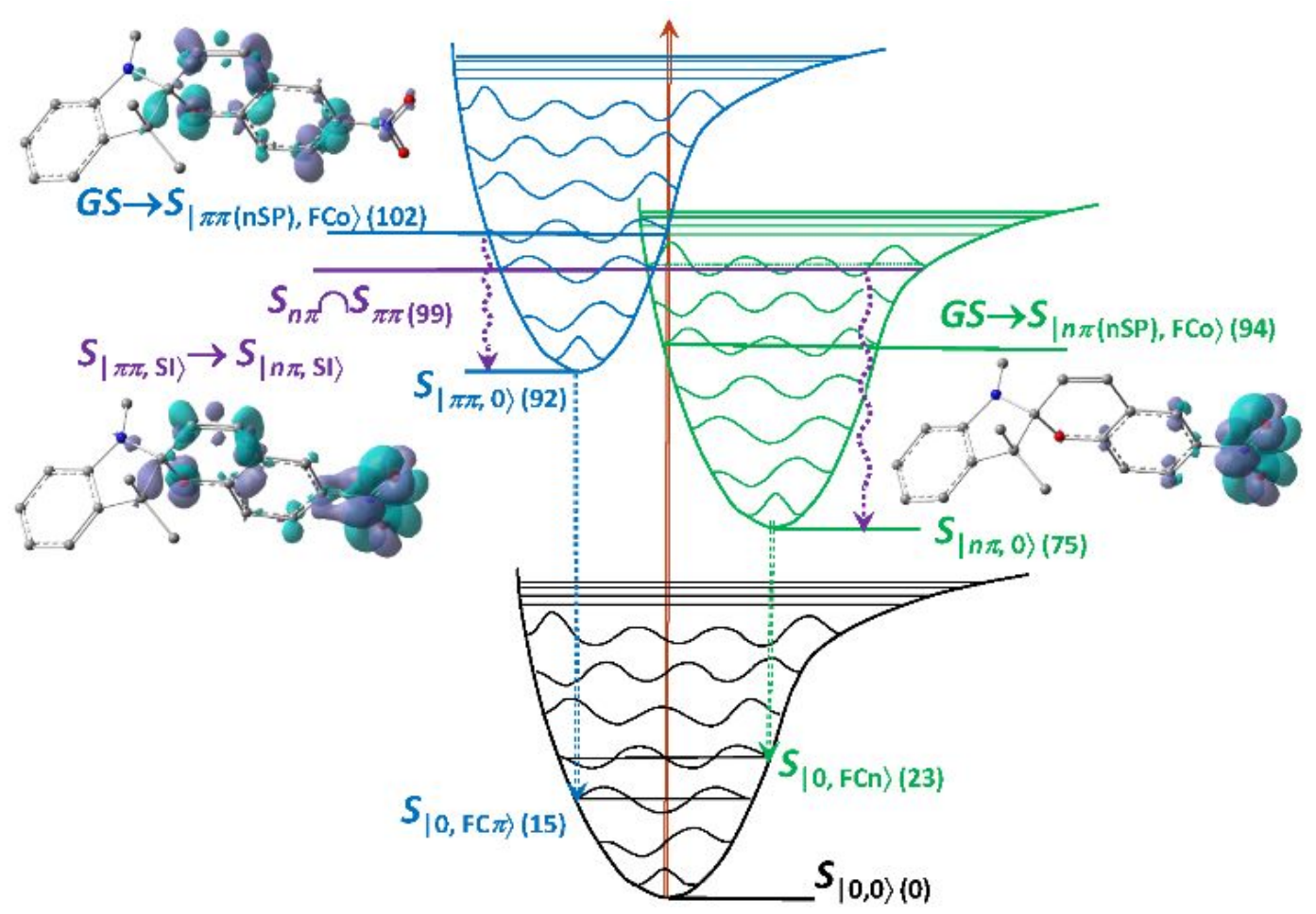

Figure 3. Sketch of vibrational PES for excited states, scheme of transitions and corresponding electron density redistribution where dark lilac space reflects positive CT of excitation and vice versa for dissipation of EDD mapping on $0.004 \mathrm{e}^{-} / \mathrm{bohr}^{3}$ isosurface throughout the text. Relative energies $(\mathrm{kcal} / \mathrm{mol})$ are presented in parenthesis. 
The photonic excitation process could also lead to a population of the lowest singlet $G S \rightarrow S_{|n \pi(\mathrm{nSP}, \mathrm{FCo})\rangle}$ state $(E=94 \mathrm{kcal} / \mathrm{mol})$ through radiative processes. However, this is a very unlikely event as the oscillator strength for the transition between $\pi \rightarrow \pi^{*}$ and $n \rightarrow \pi^{*}$ singlet states are almost forbidden due to their near different space symmetries.

The competition between radiationless vibrational transitions at the intersection of the two excited states, a state intersection (SI) $S_{|\pi \pi, \mathrm{SI}\rangle} \cap S_{|n \pi, \mathrm{SI}\rangle}$ at $E=99 \mathrm{kcal} / \mathrm{mol}$, or even to a relaxed $S_{|\pi \pi, 0\rangle}$ with $E=92 \mathrm{kcal} / \mathrm{mol}$, and the process of $\mathrm{C}_{\text {spiro }}-\mathrm{O}$ bond cleavage in the predissociation phase of the photochromic conversion was recently proposed. ${ }^{20}$ Compared to other spiropyran derivatives, nSP has an additional channel through the $n \rightarrow \pi^{*}$ state to provide the s2m conversion. Relaxation to $S_{|\pi \pi, 0\rangle}$ does not occur because s $2 \mathrm{~m}$ is much faster than fluorescence from this state to $S_{|\pi \pi, 0\rangle}$ with $E=15 \mathrm{kcal} / \mathrm{mol}$. On the other hand, $n \mathrm{SP}$ containing pyrrolidino[60]fullerene inherits the photochromic properties of the spiropyran moiety, as is confirmed by the observed nMC absorption while, in contrast, the hybrid compounds with halogenated spiropyrans do not undergo s $2 \mathrm{~m} \cdot{ }^{20} \mathrm{In}$ the case of the transition to $S_{|n \pi, \mathrm{SI}\rangle}$ and further vibrational relaxation to $S_{|n \pi, 0\rangle}$ (a long-lived state with forbidden vertical internal conversion to $S_{|0, \mathrm{FCn}\rangle}$ with $\left.E=23 \mathrm{kcal} / \mathrm{mol}\right)$, the probability of keeping this excited state along a part of the isomerization trajectory is high because of the lowest $S_{n \pi}$ state.

A similar qualitative picture as in Figure 3 can be used for the $S_{|\pi \pi, \mathrm{SI}\rangle} \rightarrow T_{|n \pi, \mathrm{SI}\rangle}$ transition with $E=86 \mathrm{kcal} / \mathrm{mol}$, which is lower than the $S_{|\pi \pi, \mathrm{SI}\rangle} \rightarrow S_{|n \pi, \mathrm{SI}\rangle}$ transition and even lower than 
the energy of $S_{|\pi \pi, 0\rangle}$ because of the nonadiabtic process. The transition energies at the respective equilibrium positions are $E=75 \mathrm{kcal} / \mathrm{mol}$ for $S_{|\mathrm{n} \pi, 0\rangle}$ and $E=62 \mathrm{kcal} / \mathrm{mol}$ for $T_{|\mathrm{n} \pi, 0\rangle}$. Vibrational dissipations of $S_{|n \pi, \mathrm{SI}\rangle}$ or $T_{|n \pi, \mathrm{SI}\rangle}$ could compete with s2m, which will be considered next.

This stationary scheme was used in order to provide an understanding of the $n \rightarrow \pi^{*}$ states in the $\mathrm{s} 2 \mathrm{~m}$ process and shows that adiabatic absorption and highly effective non-adiabatic transitions to the $n \rightarrow \pi^{*}$ states make the states from which pre-dissociation can start electronically populated. The kinetic trajectory of the photo-transformation from the excited $n \mathrm{SP}$ to $n \mathrm{MC}$, taking the $n \rightarrow \pi^{*}$ states into account, should explain the mechanism in detail, which is followed next.

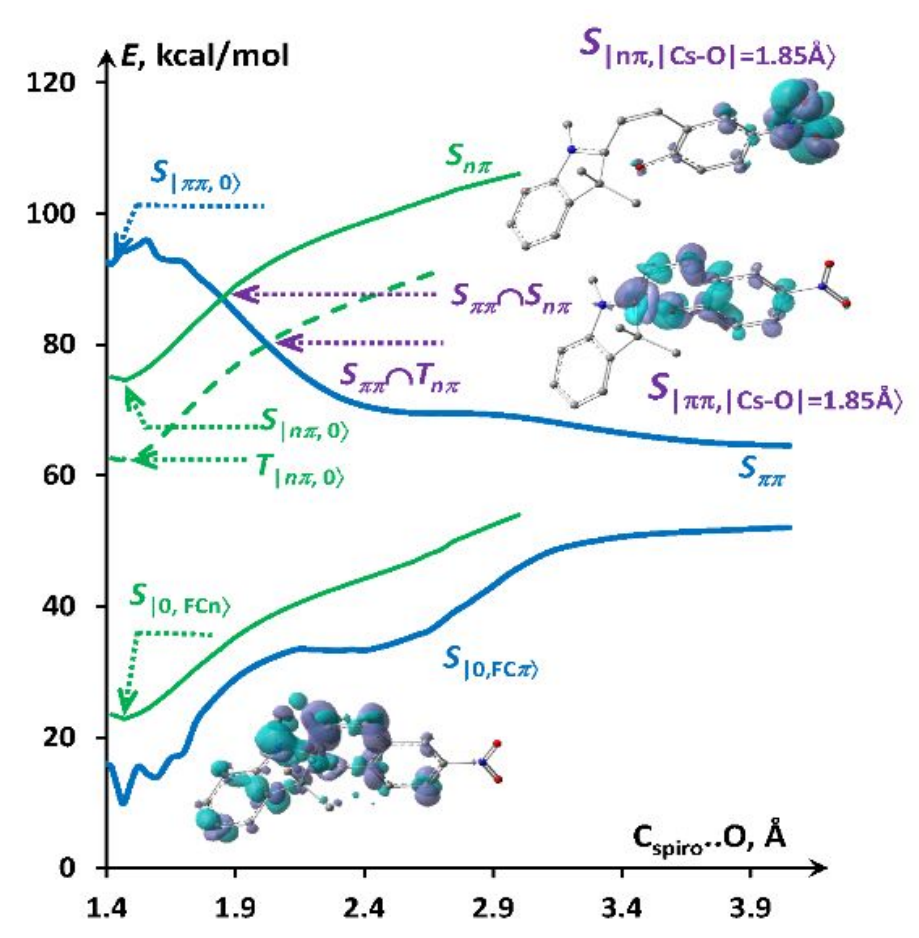

Figure 4. PES of excited states on PIT from $n$ SP to QSC through $\mathrm{C}_{\text {spiro }}-\mathrm{O}$ bond stretching for $S_{\pi \pi}$ (blue solid lines), $S_{n \pi}$ (green solid) and $T_{n \pi}$ (dashed green) optimized in ground and corresponding excited states. EDD is depictured for the SI and GS points. 
The stretching of the $\mathrm{C}_{\text {spiro }}-\mathrm{O}$ bond in combination with the optimization of the rest of the structure is one of the most probable s2m pathways after excitation (Figure 4). For each single point on the isomerization trajectories, the energy of the conformer in the corresponding excited state is obtained using the vertical transition energy from the relaxed molecular structure of the electronic ground state. The origins of the curves in Figure 4 thus coincide with the relative energies in Figure 3. The $S_{\pi \pi}$ curve is terminated at the QSC (Figure 2d and Table 2).

The elongation of the oxy-spiro bond leads to a decrease in the $S_{\pi \pi}$ energy, whereas the energies of both the singlet and triplet $n \rightarrow \pi^{*}$ states increase once the SI has been reached. Conformers with equal excited-state energies, $E=87 \mathrm{kcal} / \mathrm{mol}$ on PIT at $\left|\mathrm{C}_{\text {spiro }}-\mathrm{O}\right|=1.85$ $\AA$ of $S_{\pi \pi} \cap S_{n \pi}$, and $E=80 \mathrm{kcal} / \mathrm{mol}$ on PIT at $\left|\mathrm{C}_{\text {spiro }}-\mathrm{O}\right|=2.03 \AA$ of $S_{\pi \pi} \cap T_{n \pi}$, can easily switch the nature of the state. Figure 4 shows that the electron density difference (EDD) in the $S_{\pi \pi}$ state is delocalized over the whole $n$ SP structure (Figure 4, the lowest conformer) but is being redistributed with increasing oxy-spiro bond length, and being concentrated on the benzopyran fragment $\left(S_{|\pi \pi,[\mathrm{Cs}-\mathrm{O}]=1.85 \AA\rangle}\right)$, predominantly on the pyran ring where the nitro group is attached. In contrast, the $n \rightarrow \pi^{*}$ states are localized near the nitro substituent where the $n$ lone pair is localized $\left(S_{|n \pi,[\mathrm{Cs}-\mathrm{O}]=1.85 \AA\rangle}\right)$. The molecular structures of the different conformers are very similar and the areas of increasing electronic density (positive EDD) in the corresponding excited states are partially overlapping on the $\mathrm{C}_{5}-\mathrm{C}_{6}-\mathrm{C}_{7}$ boundary line, providing an effective channel for excited-state energy transfer facilitating the $S_{n \pi} \rightarrow S_{\pi \pi}$ or $T_{n \pi} \rightarrow S_{\pi \pi}$ transitions from the excited nitro group to the core ring. 
The $n \rightarrow \pi^{*}$ states are not dissociative, leaving only the $S_{\pi \pi}$ state as a pathway for s $2 \mathrm{~m}$ isomerization. Moreover, the stability of $n \mathrm{SP}$ allows for reversible processes if these states are relaxed. The maximum energy barrier for $S_{|\mathrm{n} \pi, 0\rangle} \leftrightarrow S_{\pi \pi} \cap S_{n \pi}$ is $12 \mathrm{kcal} / \mathrm{mol}$, which is competitive with the room temperature energy distribution, where the average thermal energy of $3 / 2 k \mathrm{~T}$ corresponds to about $1 \mathrm{kcal} / \mathrm{mol}$.

Thus, the excited-state energy transferred to the $n \rightarrow \pi^{*}$ states is brought back into the dissociative $S_{\pi \pi}$ PES along a s2m trajectory. This phenomenon, which can be interpreted as a dual energy bypass (DEB), is an advantage for hybrid compounds in order to keep the photochromic properties intact because the energy point is the lowest for the excited states of the total system.

Hybrid compounds with different bridges. A mechanism of exited-state energy exchange between $\pi \rightarrow \pi^{*}$ and $n \rightarrow \pi^{*}$ states in hybrid compounds with [60]fullerene can now be described through DEB. Two different bridges were used to design hybrid compounds of $\mathrm{PF}_{60}-\mathrm{nSP}^{20}$ (Figure 5a) and $\mathrm{EF}_{60}-\mathrm{nSP}$ (Figure 5b, SI). Several key conformers illustrate the DEB scheme for maintaining the photochromic properties in these systems.

The adiabatic absorption $G S \rightarrow S_{i \gg>1,|\pi \pi(\mathrm{nSP}), \mathrm{FCo}\rangle}$ of $E=102 \mathrm{kcal} / \mathrm{mol}$ is localized exclusively on the $n \mathrm{SP}$ moiety and coincides with that observed for isolated $n \mathrm{SP}$ in both hybrid compounds. The vibronic transitions $S_{j>1,|\pi \pi, \mathrm{SI}\rangle} \rightarrow S_{j-1>1,|n \pi, \mathrm{SI}\rangle}$ is illustrated by EDD between these states and give rise to a state with energy $E=97 \mathrm{kcal} / \mathrm{mol}$, which is higher in energy than numerous lower singlet states of [60]fullerene with bridges. Finally, the nonadiabatic transition back to the $S_{\pi \pi}$ when $\left|\mathrm{C}_{\text {spiro }}-\mathrm{O}\right|=1.85 \AA$ at an energy of $E=87 \mathrm{kcal} / \mathrm{mol}$ leads the system to the lowest singlet state of the whole system. The corresponding energies 
are the same for both compounds. This DEB trick allows photochromic properties to be inherited in compounds involving different carbon allotropes.

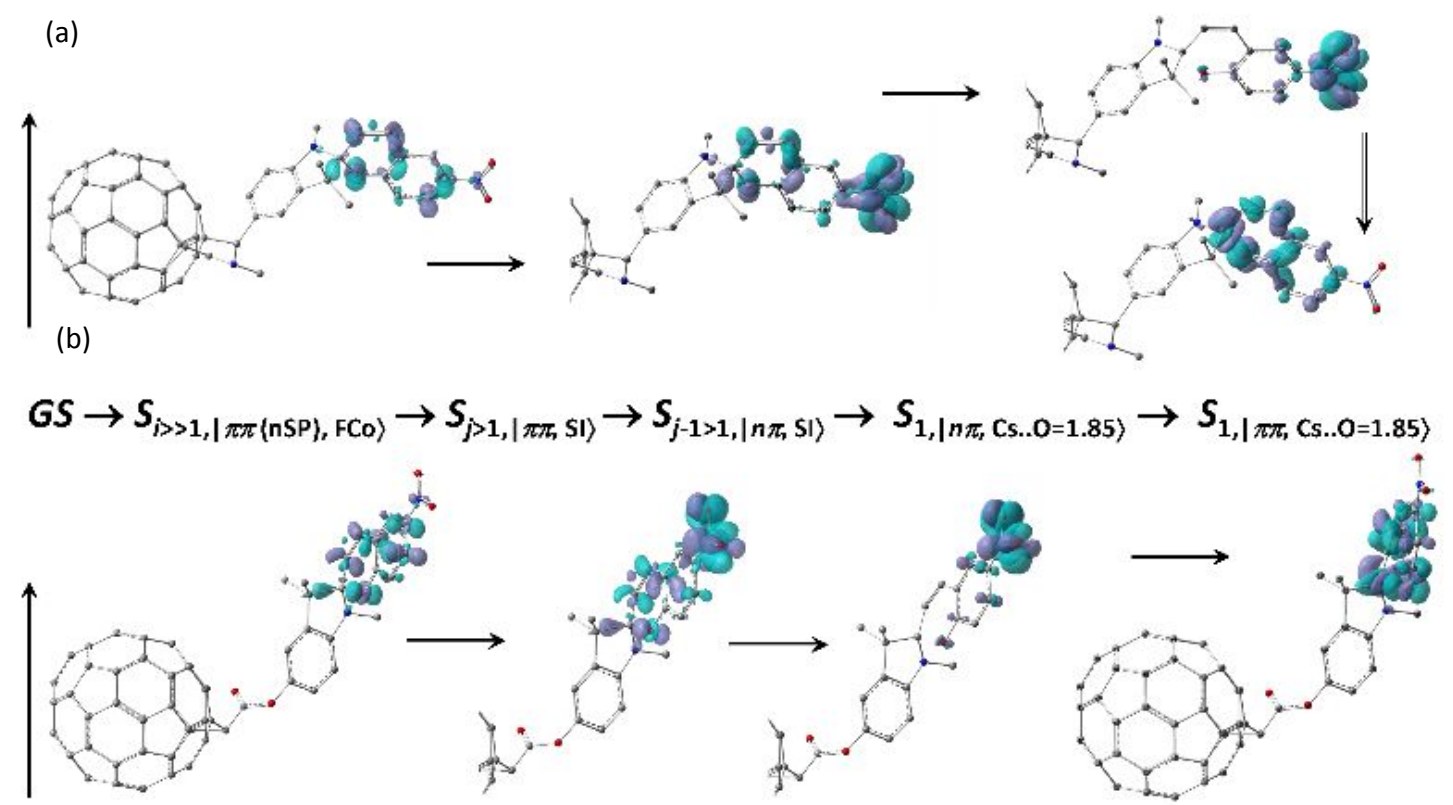

Figure 5. (a) $\mathrm{PF}_{60}-\mathrm{nSP}$ and (b) $\mathrm{EF}_{60}-\mathrm{nSP}$ conformers in points of transitions with corresponding EDDs. DEB sequential stages after vertical excitation include (from left to right) intersection with a $n \rightarrow \pi *$ state, $\mathrm{C}_{\text {spiro. }}$ O stretching and energy transfer back to $S_{\pi \pi}$.

The MOs of the excited states, EDD between states and energy levels of these states are identical to those observed in the case of the individual nSP conformers. This follows from the almost perfect decoupling between the different moieties of the hybrid compounds, preventing electronic redistribution from the photochromic fragment and overlap of MOs in these states localized on different units on the $\mathrm{s} 2 \mathrm{~m}$ trajectory compared to the isolated $n$ SP conformers. This also makes the transition probability of excited-state energy transfer to the fullerene system much smaller than the vibrational relaxation from $S_{\pi \pi}$ to $S_{n \pi}$ states in the first stage of the transformation process. Thus, the photochromic properties are inherited by these hybrid compounds on kinetic $\mathrm{s} 2 \mathrm{~m}$ trajectories. 
Thermal conversion. The discussion above established that $n \rightarrow \pi^{*}$ states play an important role in the determination of the PES along the $\mathrm{C}_{\text {spiro }}-\mathrm{O}$ bond between the equilibrium position near $1.4 \AA$ and up to $2.0 \AA$. The vibrational bond stretching significantly increases the probability of populating the dissociative $S_{\pi \pi}$ state in the pretransformation phase. Bond stretching is a trigger for further isomerization, but the trajectory of this process also depends on the $\alpha, \beta$ and $\gamma$ dihedral torsion angles that define the final merocyanine forms. Irrespective of the choice of functional and basis set, the most stable conformer is the TTC isomer.

There have been a number of studies during the last decades devoted to structural transformations occurring without light absorption. ${ }^{17,19,23,27}$ It has been assumed that the most probable thermal isomerization reactions arise from merocyanines, but the spontaneous conversion of spiropyrans has been considered theoretically as well. ${ }^{23,27}$ The conical intersection $S_{\pi \pi} / S_{0}$ method provides the isomerization actually in the electronic ground state of the corresponding intermediate conformer after deactivation of excited spiropyran. ${ }^{18,19,24}$ The isomerization trajectories, that were originated at the equilibrated spiropyran derivatives and produced by fixing the key geometric parameters $\left(\mathrm{C}_{\text {spiro. }} . \mathrm{O}\right.$ length and $\beta$ torsion) during the optimization, usually went through a QSC similar to that shown in Figure 2d.

The mechanism of the thermal conversion between $n \mathrm{SP}$ and $n \mathrm{MC}$ through the back conversion to the original unexcited $n \mathrm{SP}$ form needs to be considered. Two opposite trajectories starting from $n \mathrm{SP}$ and $n \mathrm{MC}$, respectively, were generated to establish alternative pathways with low-energy barriers for the isomerization reaction. Both ground- 
and excited-state PESs (Figure 6) are plotted because the thermal transformation can be detected experimentally by measuring spectral shifts in the absorption bands. The absorption process was calculated as a vertical excitation from the ground state to the lowest active $\pi \rightarrow \pi^{*}$ state. The changes in the absorption curves thus reflect structural changes along the isomerization pathways. The thermal conversions arise from the optimization of the ground-state $n \mathrm{SP}$ and $n \mathrm{MC}$ with fixed extended and reduced $\mathrm{C}_{\text {spiro.. }} . \mathrm{O}$ lengths, respectively.

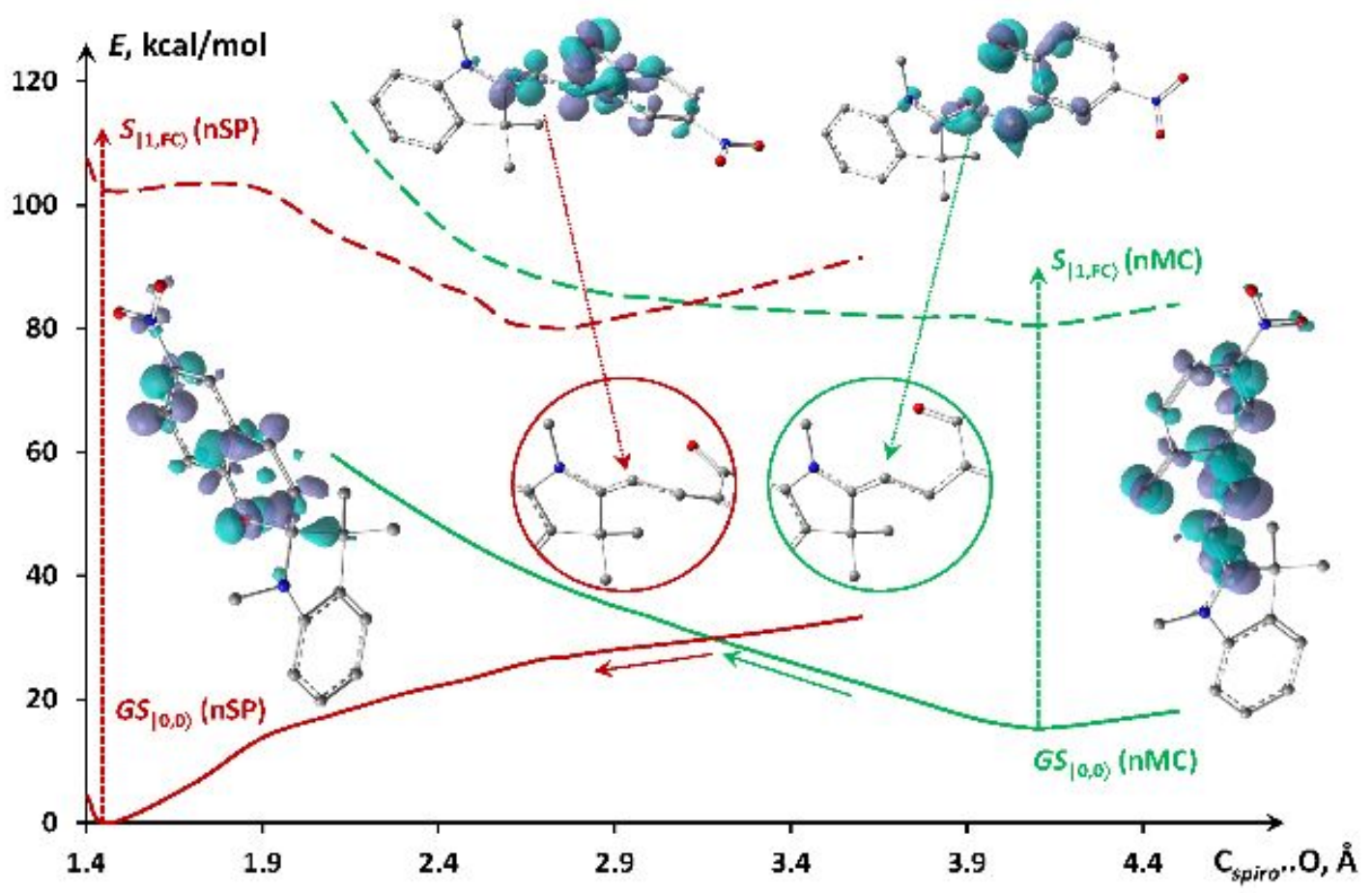

Figure 6. Opposite generated PESs of GS and absorptive $S_{\pi \pi}$ dependence on $\mathrm{C}_{\text {spiro..O }}$ length in unexcited $n \mathrm{SP}$ (in red) and $n \mathrm{MC}$ (in green) and EDD of the conformers at initial points and intersected states with structural difference between spiro units.

The benzopyran moiety with the spiro unit, which turns to a polyene bridge for $n \mathrm{MC}$, is involved in the electron density redistribution during the absorption in the $n \mathrm{SP}$ conformers 
along the isomerization trajectory, whereas there is no contribution from the other end of the molecule.

The $n \mathrm{MC}$ and $n \mathrm{SP}$ trajectories cross at $\left|\mathrm{C}_{\text {spiro }}-\mathrm{O}\right|=3.2 \AA$ with an energy barrier of 14 $\mathrm{kcal} / \mathrm{mol}$ compared to the equilibrium $n \mathrm{MC}$ structure. This "stretching" barrier is not very high considering thermal fluctuations, but the optimization process cannot find the proper pathway due to the complicate dependence of the correct geometric changes on the initial guess. For this reason, the $\gamma$ angle is instead chosen as a variable parameter in order to locate the QSC (Table 2). Another obstacle for making the transition to the $n$ SP trajectory at the barrier point is the competition of the oxygen atom with $\mathrm{C}_{\text {spiro }}$ and $\mathrm{C}_{3}$ (Figure 1 and Table 3).

Table 3. The oxygen distance and orientation torsion parameters on isomerization PES at the intersection points between ground $\left({ }^{G S}\right)$ or excited $\left({ }^{e x}\right)$ states of $n S P$ and $n M C$.

\begin{tabular}{cccccc}
\hline conf. $^{\text {param. }}$ & $\mathrm{C}_{\text {spiro. }} . \mathrm{O}$ & $\mathrm{C}_{3 . . \mathrm{O}}$ & $\alpha$ & $\beta$ & $\gamma$ \\
\hline$n S P^{e x}$ & $\mathbf{3 . 1}$ & 2.9 & -155 & 6 & 65 \\
$\mathrm{n} M C^{e x}$ & 2.4 & -175 & 101 & 4 \\
$n S P^{e x}$ & 4.2 & 3.2 & 176 & & 71 \\
$\mathrm{n} M C^{e x}$ & 2.6 & 2.5 & -157 & $\mathbf{6 0}$ & 14 \\
\hline $\mathrm{n} S P^{G S}$ & $\mathbf{3 . 2}$ & 3.1 & -169 & 42 & 23 \\
$\mathrm{n} M C^{G S}$ & & 2.5 & -163 & 123 & -20
\end{tabular}

The main difference between the conformers of the same energy at the crossing point is the values of the $\beta$ and $\gamma$ torsion angles. The distance between $\mathrm{O}$ and $\mathrm{C}_{3}$ is short enough to prevent a smooth twist, and instead the dihedral angles should flip through another "twist" barrier. This barrier can be located by performing a relaxed-structure scan through 
numerous conformers with different fixed $\beta$ and $\gamma$ torsion angles while retaining a constant oxy-spiro bond length of $3.2 \AA$. However, this is a time-consuming task because a pair of torsion values must be fixed for each single point on the trajectory and because of the very poor convergence of the optimization procedure due to the low stability of these conformers. The search for chemical reaction pathways for the isomerization is thus difficult for such large molecules with a complicated electronic structure. Smaller and simpler representative models are required for such computational techniques to work well.

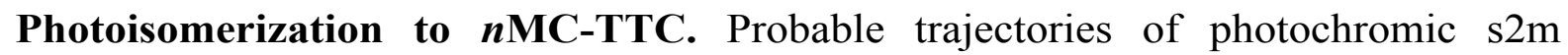
conversions are presented in Figure 7. The $n \mathrm{SP}$ curve originates at a bond length $\left|\mathrm{C}_{\text {spiro... }}\right|$ $=2.0 \AA$ and follows the dissociative excited states in Figure 4 till the QSC (Table 2) energy point. If this length is less than $3 \AA$, a full structural optimization leads to the return of this conformer to the $n \mathrm{SP}$ form. Otherwise, for oxy-spiro distances longer than $3 \AA$, the first low-energy barrier is overcome, leading to an irreversible transformation to the QSC. The QSC is an intermediate conformer toward TTC- $n \mathrm{MC}$, which is reached by a geometry optimization with a fixed torsion angle. One way to produce the $\mathrm{s} 2 \mathrm{~m}$ trajectory is to stretch the $\mathrm{C}_{\text {spiro.. }} \mathrm{O}$ to the QSC intermediate, and then to rotate around the $\beta$ bond, as the main intrinsic difference between $n \mathrm{SP}$ and $n \mathrm{MC}$ is the $\beta$ torsion angle that changes from $13^{\circ}$ to $175^{\circ}$, respectively. It was therefore natural to use this angle as a constant parameter for the optimization, starting from a value of $6^{\circ}$ for the angle at the QSC. For values of $\beta$ between $50^{\circ}$ and $70^{\circ}$, structural instabilities were observed for the conformer, with a complex dependence on the $\gamma$ torsion angle. These structural instabilities arise from the competition between $\mathrm{C}_{\text {spiro }}$ and $\mathrm{C}_{3}$ to couple with oxygen, compare for instance a bond distance of $4 \AA$ against $3.2 \AA$ at $\beta=60^{\circ}$, respectively (Table 3). This is near the point of intersection with 
the PES obtained by starting from the excited $n \mathrm{MC}$ and using the same parameter in the range from $175^{\circ}$ to $25^{\circ}$ (Figure 7) with a " $\beta$ twist" barrier equal to $8 \mathrm{kcal} / \mathrm{mol}$. At the same time, the $\gamma$ angle changes from $71^{\circ}$ in the nSP conformer to $14^{\circ}$ in the $n \mathrm{MC}$ counterpart with almost the same $\left|\mathrm{C}_{\text {spiro. }} . \mathrm{O}\right|=2.6 \AA$ and $\left|\mathrm{C}_{3} . . \mathrm{O}\right|=2.5 \AA$ bond lengths.

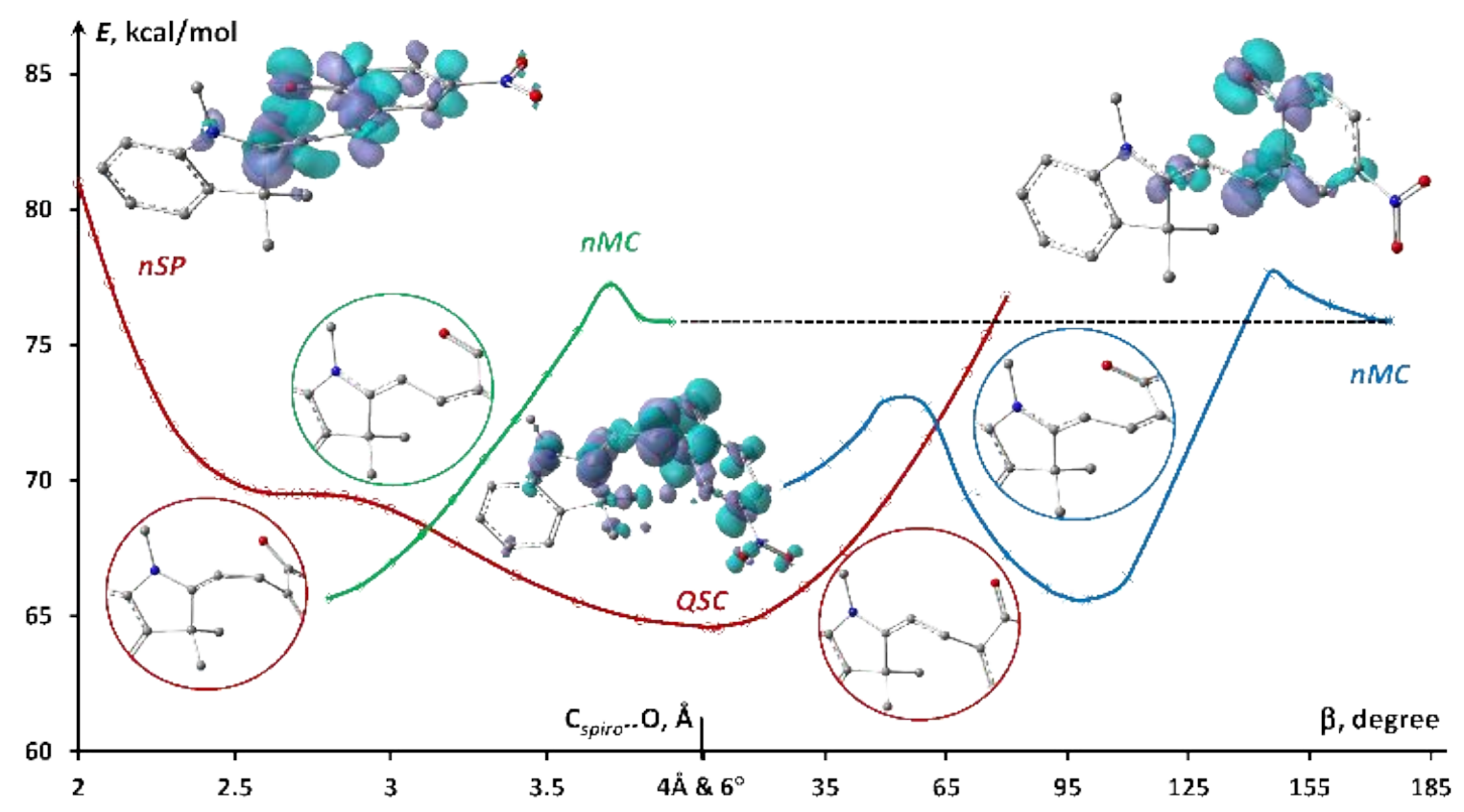

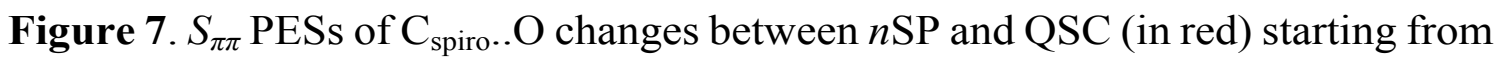
$n \mathrm{MC}$ (in green). $S_{\pi \pi}$ PESs of $\beta$ torsions starting from QSC (in red) and $n \mathrm{MC}$ (in blue). EDDs on the conformers in initial and QSC points and structural difference between spiro units at the intersection points.

A second approach is to consider thermal conversion at the intersection of the PESs of the opposite isomerization reactions of both $n \mathrm{SP}$ and $n \mathrm{MC}$ with a straightforward manipulation of the $\mathrm{C}_{\text {spiro.. }}$ O connection by elongation and reduction of the bond length. The PES of $n \mathrm{MC}$ originates from the same structure as in the case with the $\beta$ parameter. The $n \mathrm{SP}$ and $n \mathrm{MC}$ curves cross each other at $\left|\mathrm{C}_{\text {spiro.. }} \mathrm{O}\right|=3.1 \AA$, far away from the QSC. 
"Stretch" conversion to the $n \mathrm{MC}$ trajectory looks barrierless but with crucial differences in both the $\beta$ and $\gamma$ angles (Table 3). This alternative pathway avoids the QSC. The energy barriers are nearly $12 \mathrm{kcal} / \mathrm{mol}$ for the first method and $9 \mathrm{kcal} / \mathrm{mol}$ for the second method to reach the balanced $n \mathrm{MC}$ form, which is quite compatible with the thermal energy available at room temperature.

It should be noted that the transformations proceed with an electron density redistribution at the end of the molecule that contains the nitro group. Indoline plays a less important role than the rest of the molecule in these photophysical processes. This can be taken into account in the synthesis of spiro-benzopyran derivatives.

\section{CONCLUSIONS}

Kinetic photochromic and thermal isomerization trajectories for the transformation between nitro-spiropyran in its ground and excited states and its trans-trans-cis merocyanine form were generated using a sequence of constrained geometry optimizations, fixation of the $\mathrm{C}_{\text {spiro }}-\mathrm{O}$ bond length, and the $\gamma$ dihedral angle along reaction pathways on the PESs.

The role of the lowest $n \pi^{*}$ states formed on a lone pair of the nitro substituent was established in detail during the predissociative transformation of the photochromic conversion in hybrid compounds of nitro-spiropyran bonded to [60]fullerene with two different bridges. The $n \pi *$ type are not dissociative states on photo-isomerization trajectories but can accept and return an excitation from/to the low-lying dissociative 
photoactive $\pi \pi^{*}$ state at two points of intersection between their energy surfaces that provides a "dual energy bypass" in the hybrid systems.

The reversible "dual energy bypass" mechanism of photochromic inheritance in hybrid compounds of nitro-spiropyran containing [60]fullerene was described. This process includes both the adiabatic absorption from the electronic ground state to the photoactive Frank-Condon $\pi \rightarrow \pi^{*}$ state and two highly effective nonadiabatic dissipative transitions between the $n \pi^{*}$ and $\pi \pi^{*}$ states of the photochromic fragment. Such a series of transitions prevents energy loss through the carbon allotrope and keep the photochromic properties intact, because the $\pi \pi^{*}$ state is energetically lower than all other singlet excited states of the total system for transitions from the $n \pi^{*}$ state. Thus, the prediction of earlier work ${ }^{14}$ on the inheritance of the photochromic properties is confirmed by the kinetic trajectories of the $n$ SP photoisomerization process in these hybrid compounds.

\begin{abstract}
Alternative pathways of photochromic conversion and thermal transformation were produced using only the $\mathrm{C}_{\text {spiro }}-\mathrm{O}$ bond length variable as an optimization parameter for two opposite potential energy surfaces originating from equilibrated nitro-spiropyran and merocyanine, respectively.
\end{abstract}

ASSOCIATED CONTENT 
Supporting Information contains geometric parameters of the key conformers on photo- and thermo-chromic transformation trajectories between nitro-spiropyran and nitro-merocyanine molecules and their hybrid compounds with [60]fullerene.

\section{ACKNOWLEDGMENTS}

The research was supported by Basic Science Research Program through the National Research Foundation of Korea (NRF) funded by the Ministry of Education (Grant No. 2019R1I1A1A01061339). All calculations were implemented in Norway with the support of the Norwegian Supercomputer Program (Grant No. NN4654K). The work in Tromsø was supported by the Research Council of Norway through a Centre of Excellence Grant (Grant No. 262695). Pavel Avramov acknowledges National Research Foundation of Republic of Korea for support under grant NRF- 2017R1A2B4004440.

\section{REFERENCES:}

(1) Wang, L.; Li, Q. Photochromism into Nanosystems: Towards Lighting up the Future Nanoworld. Chem. Soc. Rev. 2018, 47, 1044-1097

(2) Karimi, M.; Sahandi Zangabad, P.; Baghaee-Ravari, S.; Ghazadeh, M.; Mirshekari, H.; Hamblin, M. R. Smart Nanostructures for Cargo Delivery: Uncaging and Activating by Light. J. Am. Chem. Soc. 2017, 139, 4584-4610.

(3) Klajn, R. Spiropyran-Based Dynamic Materials. Chem. Soc. Rev. 2014, 43, 148-184.

(4) Tomasulo, M.; Yildiz, I.; Raymo, F. M. Nanoparticle-Induced Transition from Positive to Negative Photochromism. Inorganica Chim. Acta 2007, 360, 938-944.

(5) Orgiu, E.; Samorì, P. 25th Anniversary Article: Organic Electronics Marries Photochromism: Generation of Multifunctional Interfaces, Materials, and Devices. Adv. Mater. 2014, 26, $1827-$ 1845.

(6) Qin, M.; Huang, Y.; Li, F.; Song, Y. Photochromic Sensors: A Versatile Approach for Recognition and Discrimination. J. Mater. Chem. C 2015, 3, 9265-9275. 
(7) Shepelenko, E. N.; Revinskii, Y. V.; Tikhomirova, K. S.; Karamov, O. G.; Dubonosov, A. D.; Bren, V. A.; Minkin, V. I. Photochromic and Fluorescent 5-Coumarinyl-4-Pyrrolylthiazoles. Mendeleev Commun. 2016, 26, 193-195.

(8) Börjesson, K.; Herder, M.; Grubert, L.; Duong, D. T.; Salleo, A.; Hecht, S.; Orgiu, E.; Samori, P. Optically Switchable Transistors Comprising a Hybrid Photochromic Molecule/n-Type Organic Active Layer. J. Mater. Chem. C 2015, 3, 4156-4161.

(9) Chibisov, A. K.; Görner, H. Photoprocesses in Spiropyran-Derived Merocyanines. J. Phys. Chem. A 1997, 101, 4305-4312.

(10) Cottone, G.; Noto, R.; La Manna, G.; Fornili, S. L. Ab Initio Study on the Photoisomers of a NitroSubstituted Spiropyran. Chem. Phys. Lett. 2000, 319, 51-59.

(11) Görner, H. Photochromism of Nitrospiropyrans: Effects of Structure, Solvent and Temperature. Phys. Chem. Chem. Phys. 2001, 3, 416-423.

(12) Futami, Y.; Chin, M. L. S.; Kudoh, S.; Takayanagi, M.; Nakata, M. Conformations of NitroSubstituted Spiropyran and Merocyanine Studied by Low-Temperature Matrix-Isolation Infrared Spectroscopy and Density-Functional-Theory Calculation. Chem. Phys. Lett. 2003, 370, 460-468.

(13) Holm, A.-K.; Mohammed, O. F.; Rini, M.; Mukhtar, E.; Nibbering, E. T. J.; Fidder, H. Sequential Merocyanine Product Isomerization Following Femtosecond UV Excitation of a Spiropyran. $J$. Phys. Chem. A 2005, 109, 8962-8968.

(14) Sheng, Y.; Leszczynski, J.; Garcia, A. A.; Rosario, R.; Gust, D.; Springer, J. Comprehensive Theoretical Study of the Conversion Reactions of Spiropyrans: Substituent and Solvent Effects. $J$. Phys. Chem. B 2004, 108, 16233-16243.

(15) Wohl, C. J.; Kuciauskas, D. Excited-State Dynamics of Spiropyran-Derived Merocyanine Isomers. J. Phys. Chem. B 2005, 109, 22186-22191.

(16) Kortekaas, L.; Chen, J.; Jacquemin, D.; Browne, W. R. Proton-Stabilized Photochemically Reversible E/ Z Isomerization of Spiropyrans. J. Phys. Chem. B 2018, 122, 6423-6430.

(17) Cottone, G.; Noto, R.; La Manna, G. Theoretical Study of Spiropyran-Merocyanine Thermal Isomerization. Chem. Phys. Lett. 2004, 388, 218-222.

(18) Marta, S.-L.; Carlos Manuel, E.; Jose, H.-R.; Luis, S.-A. Ultrafast Ring-Opening/Closing and Deactivation Channels for a Model Spiropyran-Merocyanine System. J. Phys. Chem. A 2011, 115, 9128-9138.

(19) Liu, F.; Morokuma, K. Multiple Pathways for the Primary Step of the Spiropyran Photochromic Reaction: A CASPT2//CASSCF Study. J. Am. Chem. Soc. 2013, 135, 10693-10702.

(20) Pomogaev, V. A.; Barachevsky, V. A.; Tuktarov, A. R.; Avramov, P. V.; Artyukhov, V. Y. Inheritance of Photochromic Properties of Nitro-Substituted and Halogenated Spiropyrans Containing the Pyrrolidino[60]Fullerene. J. Phys. Chem. A 2018, 122, 505-515.

(21) Tuktarov, A. R.; Khuzin, A. A.; Tulyabaev, A. R.; Venidictova, O. V.; Valova, T. M.; Barachevsky, V. A.; Khalilov, L. M.; Dzhemilev, U. M. Synthesis, Structure and Photochromic 
Properties of Hybrid Molecules Based on Fullerene $\mathrm{C}_{60}$ and Spiropyrans. $R S C A d v . \mathbf{2 0 1 6}, 6$, 71151-71155.

(22) Tuktarov, A. R.; Khuzin, A. A.; Dzhemilev, U. M. Light-Controlled Molecular Switches Based on Carbon Clusters. Synthesis, Properties and Application Prospects. Russ. Chem. Rev. 2017, 86, 474-509.

(23) Dorogan, I. V.; Minkin, V. I. Theoretical Modeling of Electrocyclic 2H-Pyran and 2H-1,4-Oxazine Ring Opening Reactions in Photo- and Thermochromic Spiropyrans and Spirooxazines. Chem. Heterocycl. Compd. 2016, 52, 730-735.

(24) Prager, S.; Burghardt, I.; Dreuw, A. Ultrafast $\mathrm{C}_{\text {Spiro }}-\mathrm{O}$ Dissociation via a Conical Intersection Drives Spiropyran to Merocyanine Photoswitching. J. Phys. Chem. A 2014, 118, 1339-1349.

(25) Ernsting, N. P.; Arthen-Engeland, T. Photochemical Ring-Opening Reaction of Indolinespiropyrans Studied by Subpicosecond Transient Absorption. J. Phys. Chem. 1991, 95, 5502-5509.

(26) Elsaesser, T.; Kaiser, W. Vibrational and Vibronic Relaxation of Large Polyatomic Molecules in Liquids. Annu. Rev. Phys. Chem. 1991, 42, 83-107.

(27) Savarese, M.; Raucci, U.; Netti, P. A.; Adamo, C.; Rega, N.; Ciofini, I. A Qualitative Model to Identify Non-Radiative Decay Channels: The Spiropyran as Case Study. Theor. Chem. Acc. 2016, $135,211$.

(28) Hobley, J.; Pfeifer-Fukumura, U.; Bletz, M.; Asahi, T.; Masuhara, H.; Fukumura, H. Ultrafast Photo-Dynamics of a Reversible Photochromic Spiropyran. J. Phys. Chem. A 2002, 106, 22652270.

(29) Frisch, M. J.; Trucks, G. W.; Schlegel, H. B.; Scuseria, G. E.; Robb, M. A.; Cheeseman, J. R.; Scalmani, G.; Barone, V.; Mennucci, B.; Petersson, G. A.; et al. Gaussian, 09, Revision D.01, Gaussian Inc., Wallingford CT, 2013.

(30) Chai, J.-D.; Head-Gordon, M. Systematic Optimization of Long-Range Corrected Hybrid Density Functionals. J. Chem. Phys. 2008, 128, 084106.

(31) Chai, J.-D.; Head-Gordon, M. Long-Range Corrected Hybrid Density Functionals with Damped Atom-Atom Dispersion Corrections. Phys. Chem. Chem. Phys. 2008, 10, 6615.

(32) Dunning, T. H. Gaussian Basis Sets for Use in Correlated Molecular Calculations. I. The Atoms Boron through Neon and Hydrogen. J. Chem. Phys. 1989, 90, 1007-1023.

(33) Christiansen, O.; Koch, H.; Jørgensen, P. The Second-Order Approximate Coupled Cluster Singles and Doubles Model CC2. Chem. Phys. Lett. 1995, 243, 409-418.

(34) Shiozaki, H. Molecular Orbital Calculations for Acid Induced Ring Opening Reaction of Spiropyran. Dyes Pigments 1997, 33, 229-237.

(35) Laptev, A.; Lukin, A.; Belikov, N.; Fomin, M.; Zvezdin, K.; Demina, O.; Barachevsky, V.; Varfolomeev, S.; Shvets, V.; Khodonov, A. Polyenic Spirobenzopyrans: Synthesis and Study of Photochromic Properties. J. Photochem. Photobiol. Chem. 2011, 222, 16-24. 
TOC Figure:
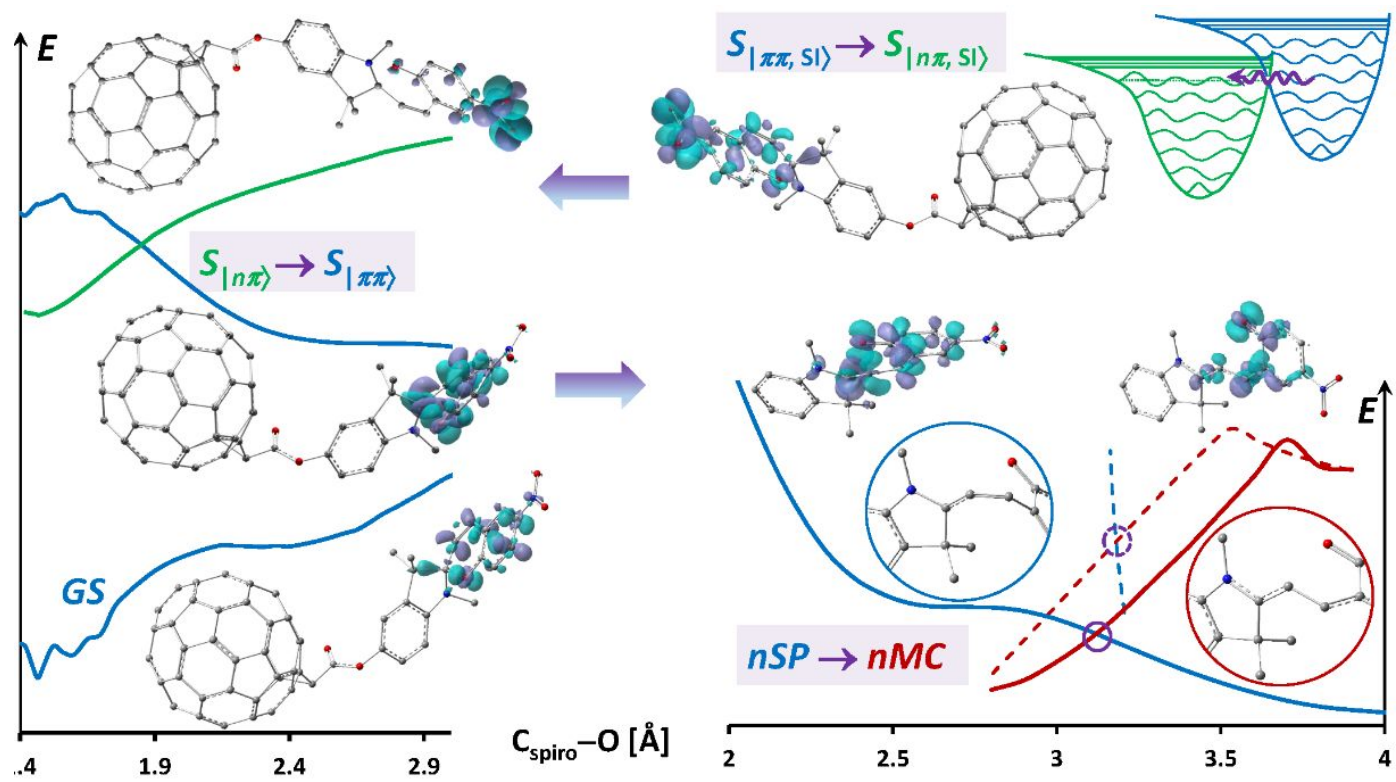

Table of Contents Graphic 

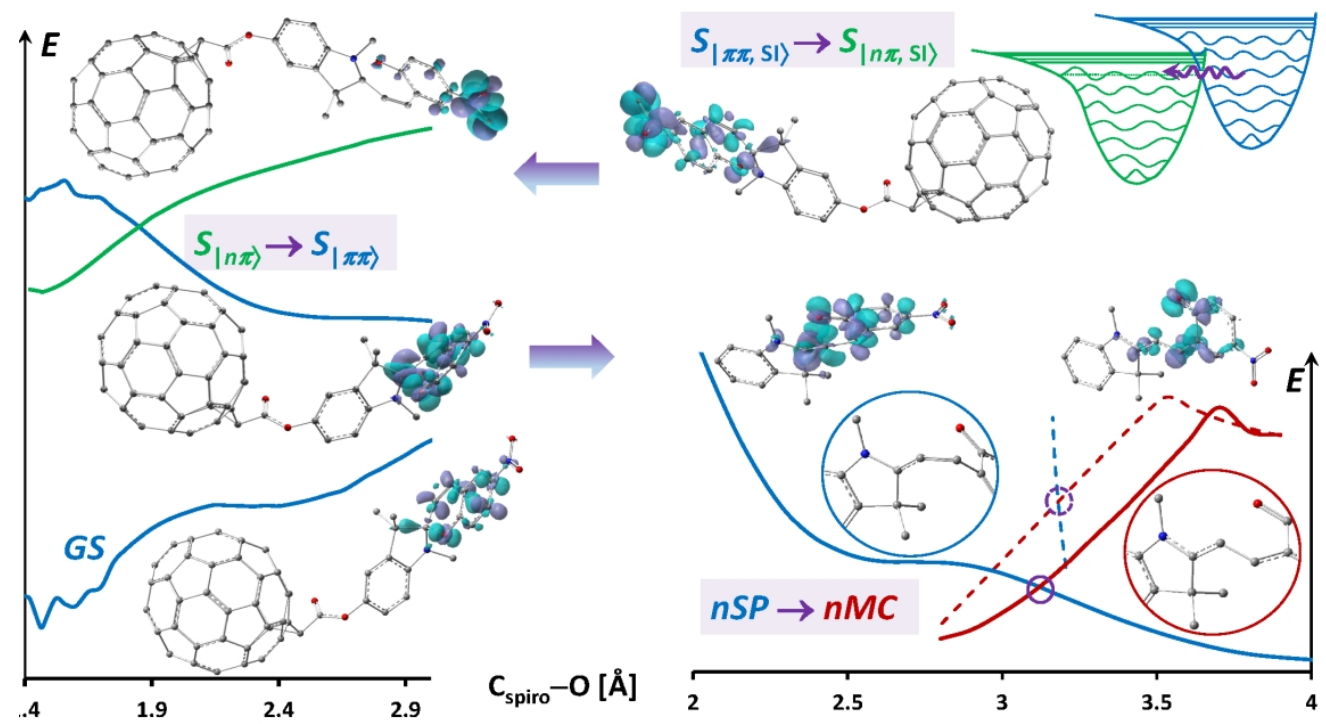

Table of Contents Graphic TOC

$338 \times 190 \mathrm{~mm}(160 \times 157 \mathrm{DPI})$ 


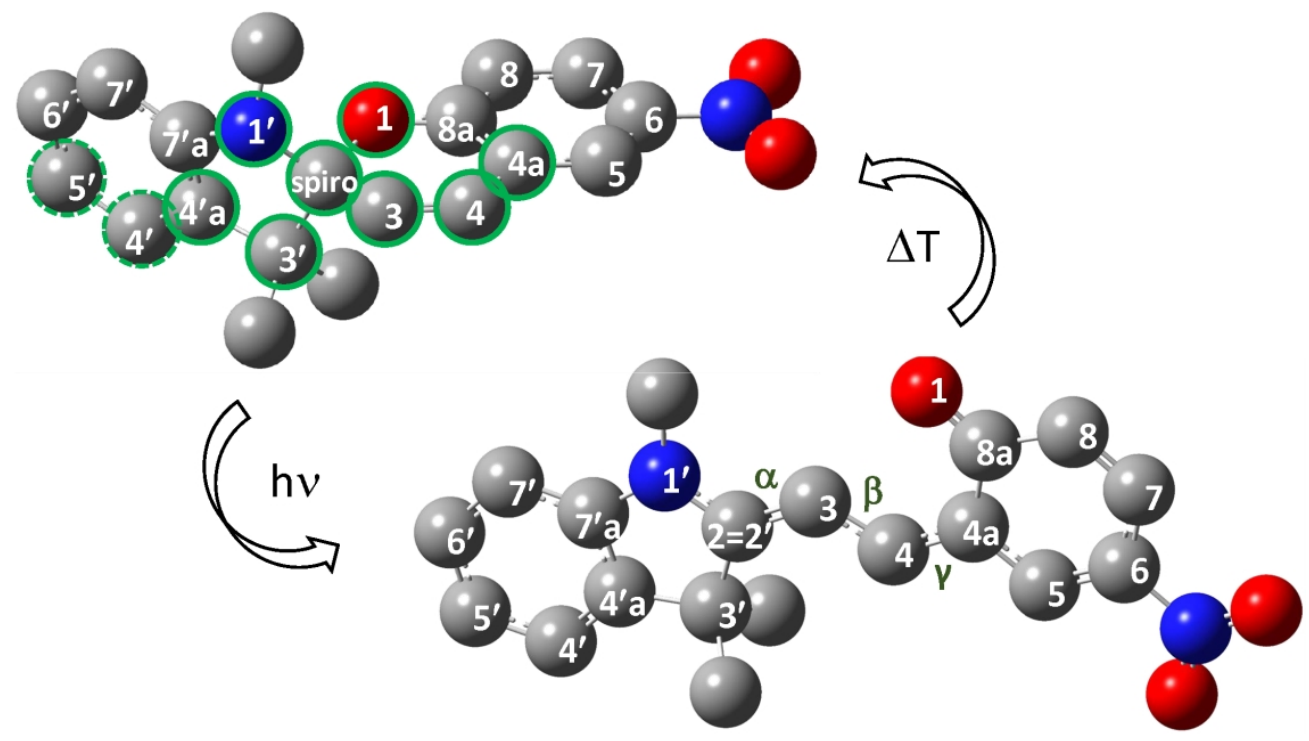

Figure 1. Structure and accepted standard numbering of nSP (a) and nMC (b) where green circle lines denote atoms which contribute the most to the difference between the ground and excited configurations. Dashed green lines in (a) define the bond about which the sequential torsions are being explored.

\section{$338 \times 190 \mathrm{~mm}(168 \times 168 \mathrm{DPI})$}



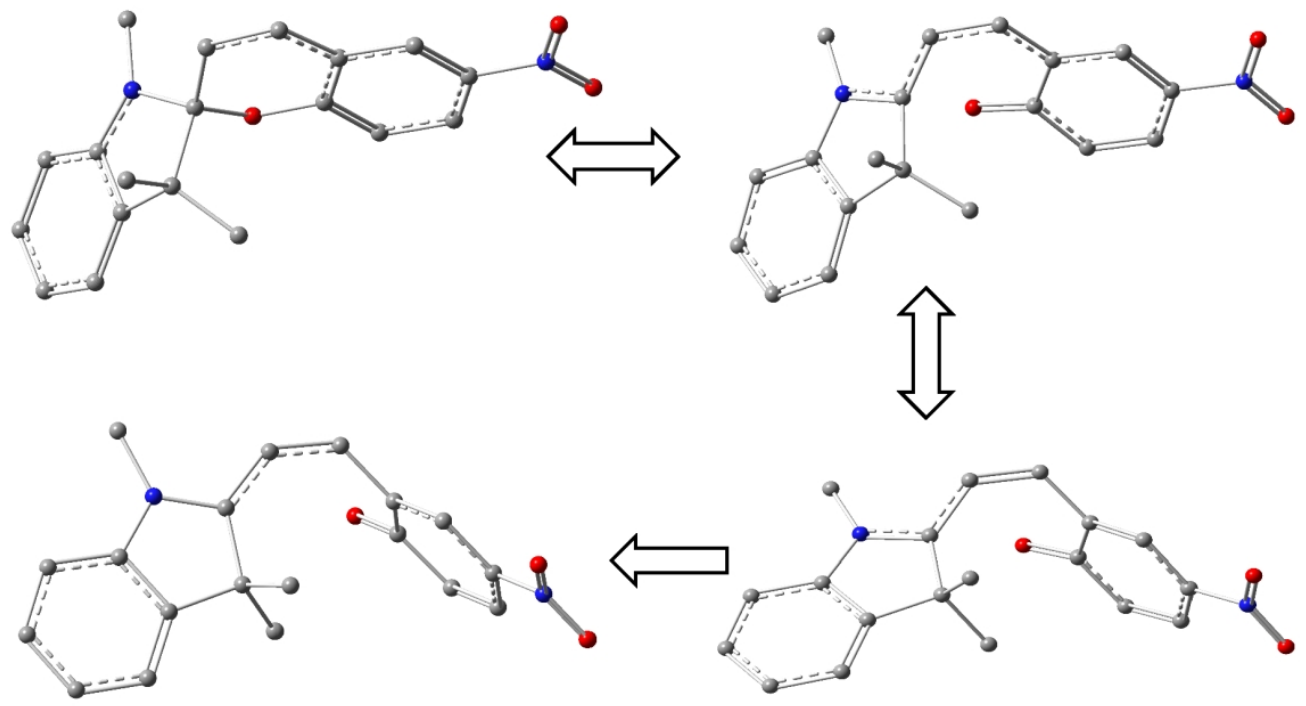

Figure 2. $\mathrm{C}_{\text {spiro.. }} \mathrm{O}$ distance elongation trajectory from equilibrium structure (a) optimized to QSC in $\mathrm{S}_{\text {пп }}$ with the length $=4.05 \AA$ (b) through an intermediate conformer with fixed $\mid$ Cspiro $\square \mathrm{O} \mid=2.00 \AA$ (c) to the energy barrier of no-return point $\mid$ Cspiro.. $0 \mid=3.00 \AA$ (d).

$338 \times 190 \mathrm{~mm}(139 \times 139 \mathrm{DPI})$ 
Figure 3. Sketch of vibrational PES for excited states, scheme of transitions and corresponding electron density redistribution where dark lilac space reflects positive CT of excitation and vice versa for dissipation of EDD mapping on $0.004 \mathrm{e} / \mathrm{bohr} 3$ isosurface throughout the text. Relative energies $(\mathrm{kcal} / \mathrm{mol}) \mathrm{are}$ presented in parenthesis.

\section{$338 \times 190 \mathrm{~mm}(200 \times 200 \mathrm{DPI})$}




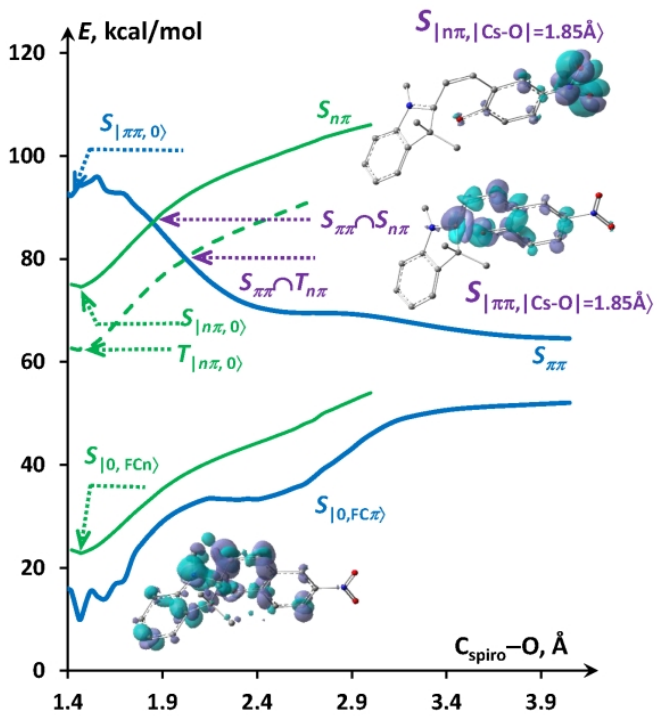

Figure 4. PES of excited states on PIT from nSP to QSC through Cspiro $\square$ O bond stretching for Snח (blue solid lines), Snח (green solid) and Tnп (dashed green) optimized in ground and corresponding excited states. EDD is depictured for the SI and GS points.

$338 \times 190 \mathrm{~mm}(200 \times 200$ DPI $)$ 
Figure 5. (a) PF60-nSP and (b) EF60-nSP conformers in points of transitions with corresponding EDDs. DEB sequential stages after vertical excitation include (from left to right) intersection with a $n \rightarrow \Pi^{*}$ state, Cspiro..O stretching and energy transfer back to Snп.

$338 \times 190 \mathrm{~mm}(220 \times 220 \mathrm{DPI})$ 


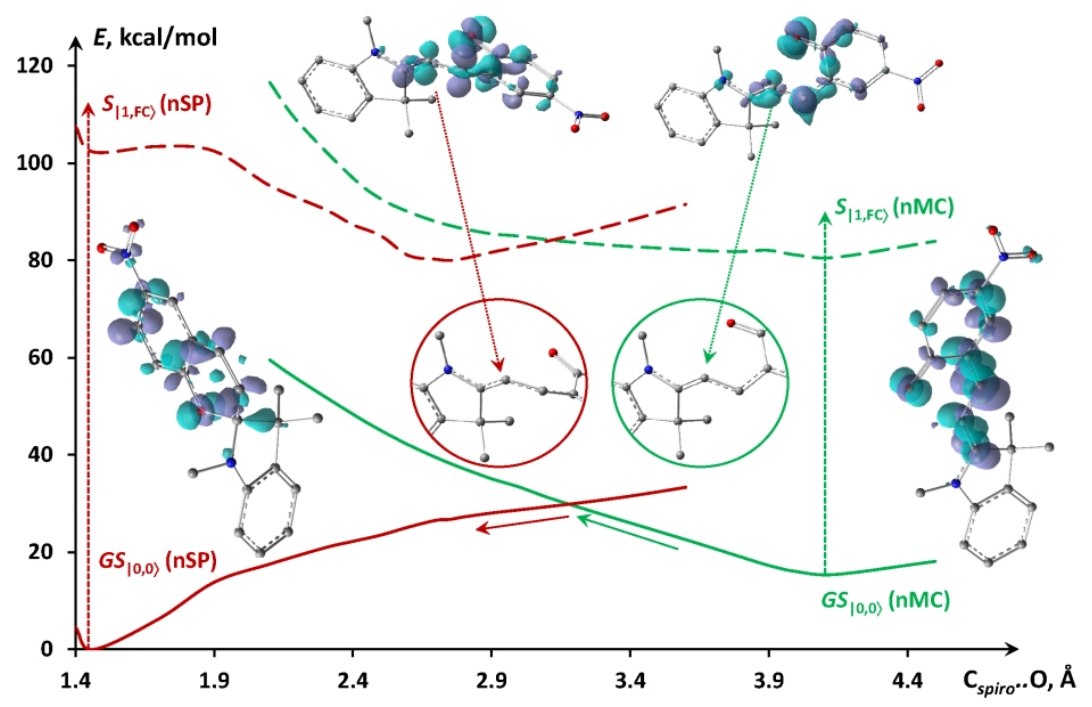

Figure 6. Opposite generated PESs of GS and absorptive Snח dependence on Cspiro..O length in unexcited nSP (in red) and nMC (in green) and EDD of the conformers at initial points and intersected states with structural difference between spiro units.

\section{$338 \times 190 \mathrm{~mm}(200 \times 200$ DPI $)$}




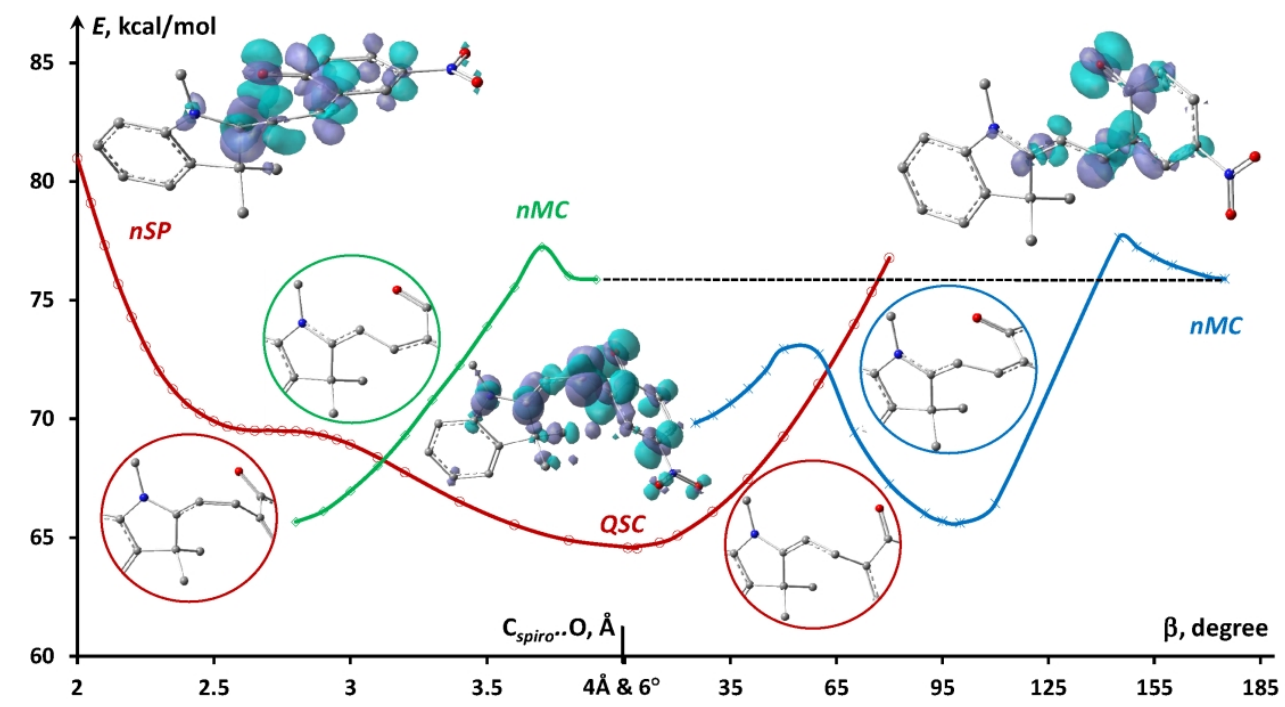

Figure 7. Sпп PESs of Cspiro.. O changes between nSP and QSC (in red) starting from nMC (in green). Snп PESs of $\beta$ torsions starting from QSC (in red) and $n M C$ (in blue). EDDs on the conformers in initial and QSC points and structural difference between spiro units at the intersection points.

$338 \times 190 \mathrm{~mm}(200 \times 200$ DPI $)$ 